\title{
Transfer Printing of Conductive Thin-Films on PDMS with Soluble Substrates for Flexible Biosensors ${ }^{\dagger}$
}

\author{
Steffen Hadeler *, Sebastian Bengsch, Maren S. Prediger and Marc Christopher Wurz \\ Institute of Micro Production Technology, Leibniz Universität Hannover, Garbsen, Germany \\ * Correspondence: hadeler@impt.uni-hannover.de \\ + Presented at the 7th International Electronic Conference on Sensors and Applications, 15-30 November \\ 2020; Available online: https://ecsa-7.sciforum.net/.
}

Published: 15 November 2020

\begin{abstract}
The resolution of commercially available electrocorticography (ECoG) electrodes is limited due to the large electrode spacing and therefore allows only a limited identification of the active nerve cell area. This paper describes a novel manufacturing process for neural implants with higher spatial resolution combining micro technological processes and PDMS as the flexible, biocompatible material. The conductive electrode structure is deposited on a water-soluble transfer substrate by PVD processes. Subsequently, the structure is contacted. Finally, the transfer to PDMS and dissolution of the transfer substrate takes place. In this way, high-resolution conductive structures can be produced on the PDMS. Transferred gold structures exhibit higher adhesion and conductivity than transferred platinum structures. The adhesion was improved by applying a silica surface modification to the conductive layer prior to transferring. Furthermore, the conductive layer is flexible, conductive up to an elongation of $10 \%$, and resistant to sodium chloride solution, mimicking brain fluids. Using the introduced production process, an ECoG electrode was manufactured and characterized for its functionality in an electrochemical impedance measurement. Furthermore, the electrodes are flexible enough to adapt to different shapes. The transfer process can also be carried out in a 3-dimensional mold to produce electrodes tailored to the individual patient.
\end{abstract}

Keywords: biosensor; ECoG; patient specific; sputtering; Sylgard 184

\section{Introduction}

The monitoring of neurophysiological processes is not only important for the localization of damaged nerve cell areas in various diseases like epilepsy [1]. The so-called brain-computer interface also aims to establish communication between the nerve cells in the brain and a computer, for example to control a prosthesis [2]. For the measurement of nerve cell activity, electroencephalography (EEG) or electrocorticography (ECoG) are the usual methods of measurement, whereby ECoG constitutes an invasive version of EEG. In ECoG, the electrodes used to measure nerve cell activity are surgically implanted below the cranial bone. Thus, the signal quality (higher sensitivities) and spatial resolution is significantly improved compared to EEG [3]. However, commercially available ECoG electrodes feature distances between the electrode contacts of about 10 $\mathrm{mm}$, which limits the minimum possible resolution. Several international research groups are researching ECoG electrodes with reduced electrode spacing and an increased number of electrodes to improve the resolution of ECoG. ECoG electrodes described in literature are produced mostly by micro technology manufacturing processes, usually using a stack of layers on a polyimide substrate [e.g., [4-8]]. On one hand, these manufacturing processes are very complex and a clean room is 
required, on the other hand, the materials used do not have the same long-term clinical experience as those of commercial electrodes [2].

Another research approach is the patient-specific adaptation of the ECoG electrode to the topography of the brain, in order to improve the signal quality of the ECoG measurement. Morris et al. use a mold manufactured by additive manufacturing, into which the electrode contacts are manually inserted [9].

In this paper, a new manufacturing approach for ECoG electrodes is presented, which is based on micro technological manufacturing processes like sputtering while using materials with long-term clinical experience such as PDMS. The chosen approach enables highly flexible and patient-specific ECoG electrodes with reduced manufacturing costs.

\section{Methods}

\subsection{Fabrication}

The manufacturing process presented in this publication is based on a transfer method developed at the Institute of Micro Production Technology (Hanover, short: IMPT), and shown schematically in Figure 1. It is based on the transfer of a thin film from a water-soluble carrier substrate to the final substrate. The carrier substrate, consisting of a water-soluble poly vinyl alcohol (PVA) film, is firstly coated in the desired configuration by means of cathode sputtering (Figure 1a). The geometrical definition of the coating is done by masking the substrate. The thin film on the carrier substrate is then contacted for evaluation using a conductive epoxy of the type CW 2400 (CircuitWorks), which is applied to the conductive coating on the PVA, as well as on the copper wires (Figure 1b). A compound of PDMS and silver particles, analogous to Larmagnac et al., can also be used for contacting with a supply line to improve the flexibility [10]. The assembly of coated and contacted PVA carrier substrate is then encapsulated in a casting mold with PDMS (Figure 1c). For this purpose, Sylgard ${ }^{\mathrm{TM}} 184$ is cured at $50^{\circ} \mathrm{C}$ overnight. The conductive coating is transferred to the PDMS and the PVA carrier substrate can be dissolved with water (Figure 1d). This results in a completely encapsulated contacting material and a body of PDMS from which only the PVD coating and the lead are accessible and in contact with the biological tissue (Figure 1e). Certain areas of the PVD coating can also be encapsulated by applying further PDMS for insulation (Figure 1f).
a) PVD-Coating of PVA
b) Bonding
c) Embedding into PDMS

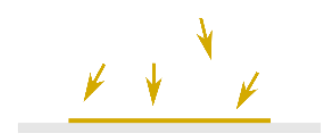

d) Curing of PDMS

Dissolving of PVA

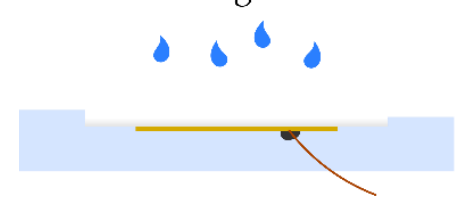

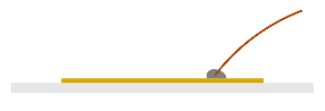

e) Contacted Layer in PDMS

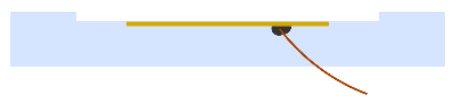

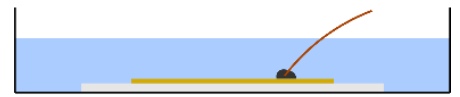

f) Isolation

Figure 1. Fabrication process: PVD coating of the transfer substrate (a), contacting (b), embedding in PDMS (c), curing the PDMS and dissolution of the transfer substrate (d) contacted, conductive structure in PDMS (e), insulation (f).

\subsection{Adhesion}

To evaluate the adhesion of the transferred layer, samples with the coating materials gold and platinum are prepared and a cross-cut test performed according to DIN EN ISO 2409. In addition, the influence of a silicatization is examined with the surface silicatization device NanoFlame NF02 (Poyltec PT GmbH), which, according to Stieghorst et al., increases the layer adhesion between PDMS 
and polyimide [11]. For this purpose, the PVA films coated with gold or platinum are silicated according to the data sheet. To reduce the influence of temperature, the flame is spread over the surface and the treatment is repeated in short intervals. The enclosed test ink was used to verify surface silicatization.

\subsection{Conductivity}

Transferred layers are evaluated with a four-wire measurement. It is also evaluated, to which extent the transfer of the thin film changes the inherent conductivity of the layer. Due to the results of the adhesion of the transferred layers, this test is carried out exclusively with gold of different layer thicknesses.

\subsection{Flexibility}

The developed manufacturing approach offers an advantage compared to commercial electrodes concerning a higher flexibility due to the missing rigid electrode contacts and leads. The flexibility of the layer is evaluated in a tensile test and a fatigue test. In the former, tensile specimens are made from PDMS according to DIN 53504 with a gold coating of different layer thicknesses and the conductivity is recorded as a function of strain. Furthermore, it is investigated whether the silicatization property, which improves the layer adhesion, also has an influence on the flexibility of the layer.

\subsection{Impedance Measurement}

The functionality of electrode samples was investigated in an electrochemical impedance measurement. The measurement procedure is based on the publication by Nahvi et al. [12].

For this measurement, samples with a layer thickness of $125 \mathrm{~nm}$ gold consisting of two electrode contacts with a size of $1 \times 1 \mathrm{~mm}$ and leads to a contacting area with a width of $250 \mu \mathrm{m}$ are prepared. In the contacting area, the connection is made with a lead and conductive epoxy. Except for the electrode contacts, the entire structure is insulated with PDMS.

\subsection{Demonstrator}

Based on the previously presented electrodes for the impedance measurement and the design in various publications, such as Kim et al. or Yan et al., an ECoG electrode was manufactured according to the process shown in Figure $1[5,13]$. The layout features eight electrode contacts with a size of $1 \times 1$ $\mathrm{mm}$. A mask is made for PVD coating.

\subsection{Adaptivity}

The presented manufacturing process can be used to pursue patient-individual adjustment. To demonstrate, analogous to Morris et al., a mold was manufactured additively, in which the coated PVA carrier substrate is inserted and encapsulated with PDMS [9]. This results in electrodes that have the same shape as the mold. The approach is firstly evaluated for a simple corrugated structure and secondly for a mold simulating brain convolutions.

\section{Results and Discussion}

\subsection{Adhesion}

In this test, the poor transfer behavior of platinum in comparison to gold is the most striking feature. In addition, almost the entire transferred platinum coating can be removed with the adhesive strip. With gold, on the other hand, the adhesion of the transferred layer is significantly better. Only a small portion of the gold layer is removed when the test is performed.

With the surface silicatization before transfer, a significant improvement in the layer adhesion is observed. However, with the coating material platinum, problems continue to occur during the transfer from the PVA substrate to the PDMS. 


\subsection{Conductivity}

The measured conductivities of the gold structures on the substrates show a dependence on the layer height. Whereby this influence is no longer significant at the highest tested layer height. Figure 2 shows the measurement results.

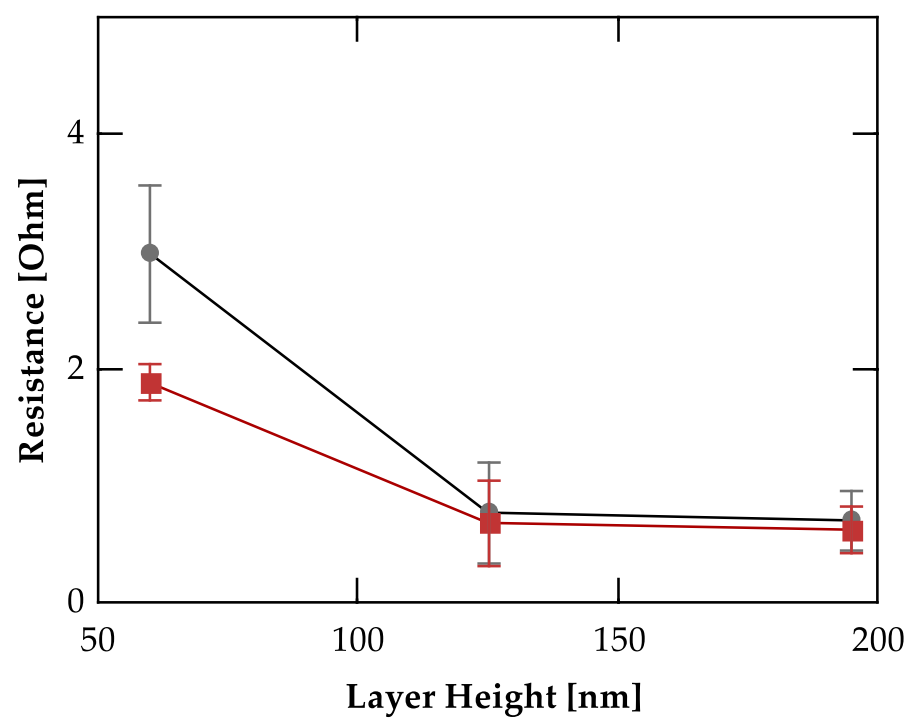

Figure 2. Resistivity as a function of film thickness, before (grey) and after (red) transfer to PDMS.

Only a minimal increase in conductivity is measured due to the transfer from the PVA to the PDMS. This difference is mainly present at the lowest tested layer height. This results in an almost complete transfer of the conductive layer from the PVA to the PDMS without affecting the layer integrity.

\subsection{Flexibility}

When testing the flexibility, a difference in the various layer thicknesses is measured. Tensile samples with a gold layer of $60 \mathrm{~nm}$ can be stretched by about $5 \%$ while maintaining conductivity, whereas those with $125 \mathrm{~nm}$ or $195 \mathrm{~nm}$ can be stretched up to $10 \%$. As shown in Figure 3, silicatization does not improve the flexibility of the transferred coating.

Microscopic cracks are apparent in the conductive layer coating because of elongation. Conductivity and thus percolation is only possible up to a certain distance of the cracks. A return to the initial state allows the formation of a continuous conductive structure without defects or flaking.

\subsection{Impedance Measurement}

The electrodes placed in a sodium chloride solution $(9 \mathrm{mg} / \mathrm{l})$ are measured with an LCR meter HM8118 (Hameg Instruments $\mathrm{GmbH}$ ) in the frequency range from $20 \mathrm{~Hz}$ to $100 \mathrm{kHz}$ at a voltage of $1 \mathrm{~V}$. The electrode used for the impedance measurement and the measured impedances as a function of frequency are shown in Figure 4. The measured impedances are in a similar order of magnitude to those found in literature. 


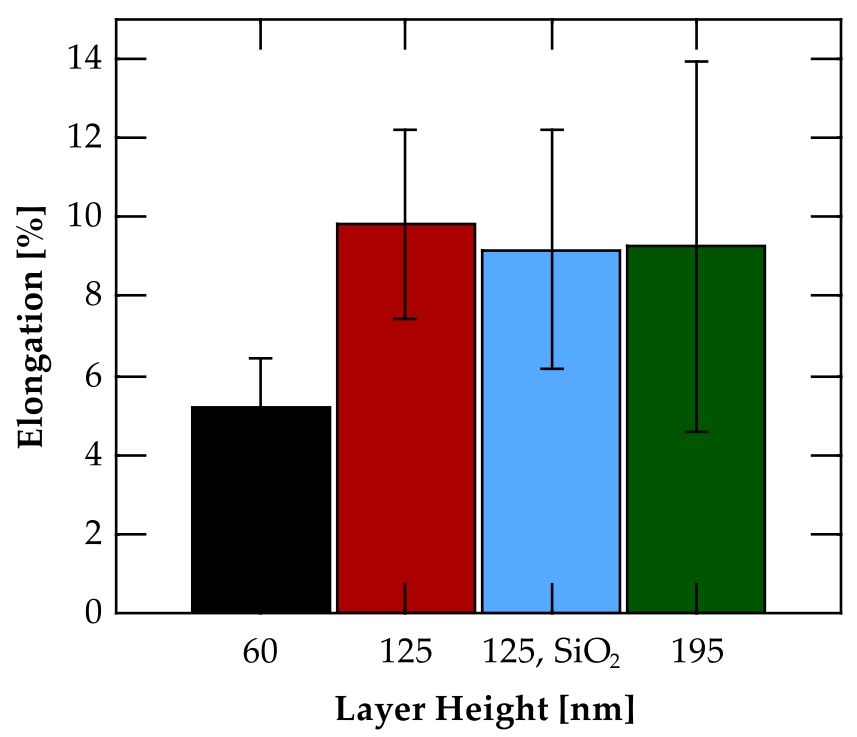

Figure 3. Elongation while maintaining conductivity as a function of the layer height and the performed silicatization.
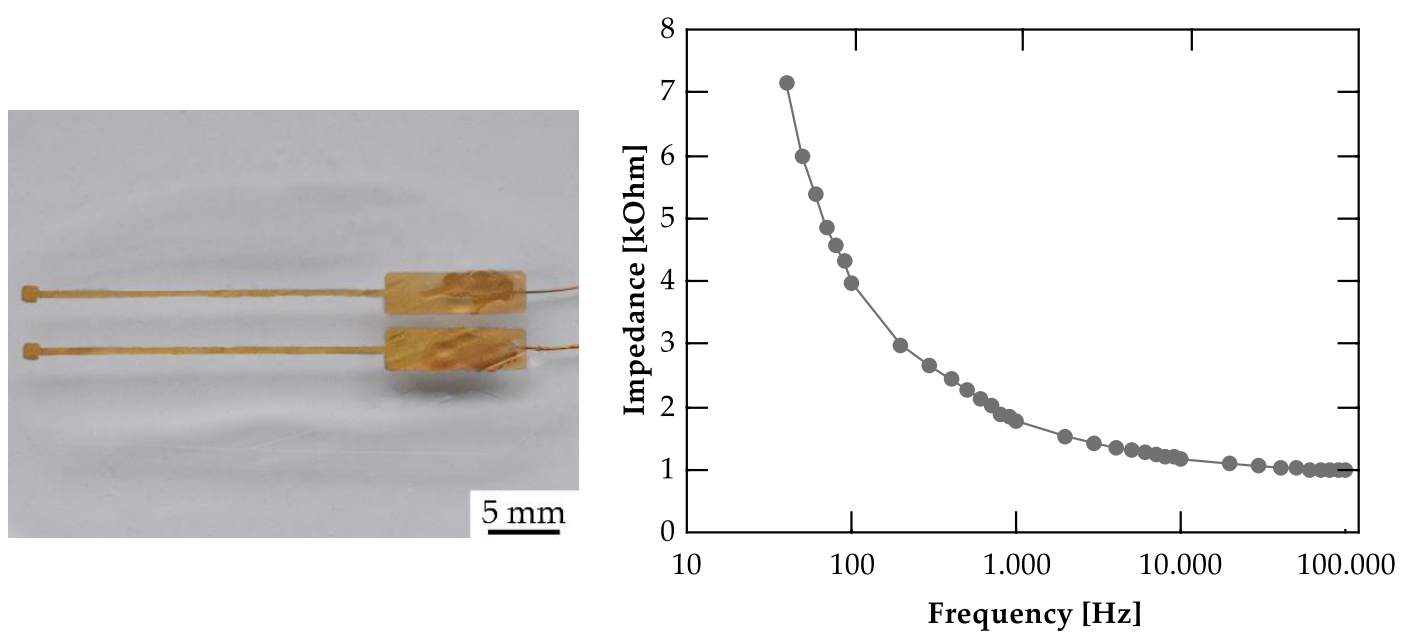

Figure 4. The left illustration shows the setup consisting of two electrode contacts. The right figure shows the measured impedances as a function of frequency.

\subsection{Demonstrator}

An example of a demonstrator with eight electrode contacts is shown in Figure 5. The electrode gap and electrode spacing is significantly smaller than commercially available electrodes.

\subsection{Adaptivity}

In both cases, the simple corrugated structure and the mold simulating brain convolutions, a complete adaptation of the carrier substrate to the shape of the mold as well as error-free transfer of the conductive gold layer to PDMS is possible. Figure 5 shows a shaped electrode with eight electrode contacts. This simple manufacturing process offers potential for cost-effective patient-specific production. 

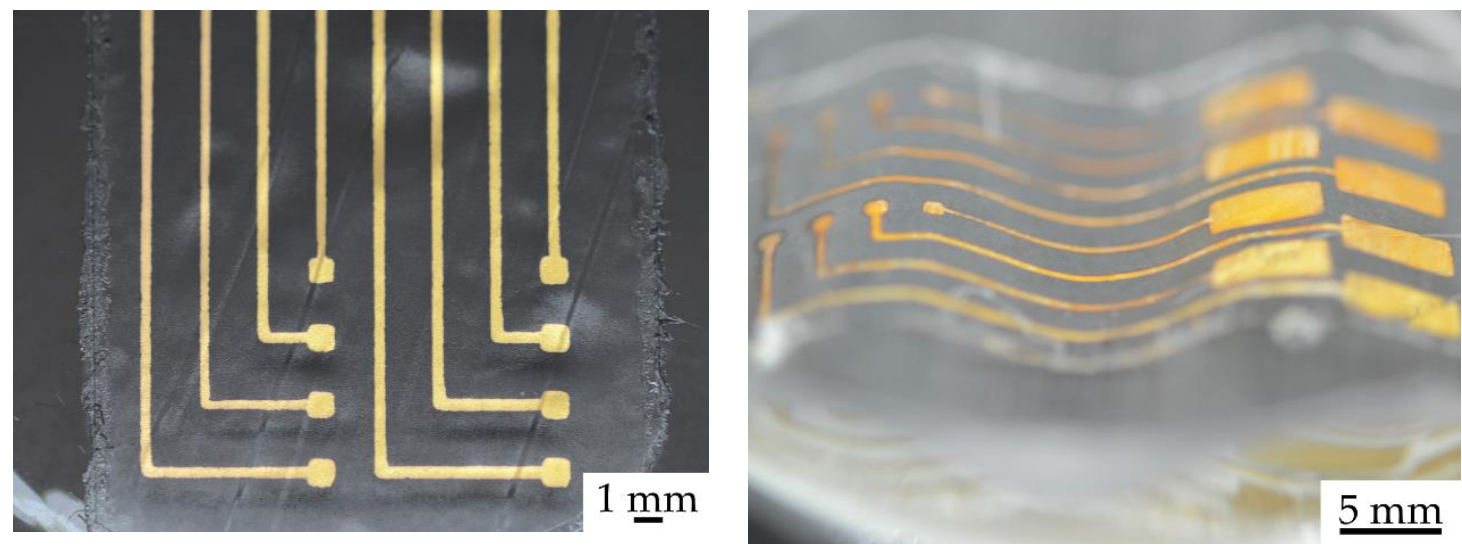

Figure 5. Manufactured ECoG demonstrator with eight electrode contacts (left) and electrode adapted to a wavy shape in PDMS (right).

\section{Conclusions}

In this paper, it was shown that a simple patient-specific production of ECoG electrodes with micro technical production methods and clinically proven materials such as PDMS is possible. This technique can be further improved by a reduction of the electrode distance or an optimization of the insulation. In addition, the electrodes can be scaled down to increase the number of electrode contacts. The transfer behavior of a stack of layers will be studied in the near future. To further increase the productivity of the manufacturing process, a so-called roll-to-roll process can be used to coat the PVA carrier substrate. Moreover, possible improvements are the direct integration of the evaluation electronics by embedding them in the PDMS, so that the implant can be fully implanted and does not leave any openings to the brain susceptible to infections. This manufacturing process can be adapted for the use with other implants or biosensors and is subject of future research at the IMPT.

Author Contributions: S.H.: Conceptualization, Investigation, Writing original draft and lead author; S.B.: Supervision; M.S.P.: Supervision and review; M.C.W.: Project administration. All authors have read and agreed to the published version of the manuscript.

Funding: This research received no external funding.

Conflicts of Interest: The authors declare no conflict of interest.

\section{References}

1. Baura, G.D. Medical Device Technologies: A Systems Based Overview Using Engineering Standards; Elsevier: Amsterdam, The Netherlands; Academic Press: Amsterdam, Waltham, MA, USA, 2012; ISBN 9780123749765 .

2. Allison, B.Z.; Dunne, S.; Leeb, R.; Del R. Millán, J.; Nijholt, A. Towards Practical Brain-Computer Interfaces. Bridging the Gap from Research to Real-World Applications; Springer: Berlin/Heidelberg, Germany, 2013; ISBN 978-3-642-29745-8.

3. Ball, T.; Kern, M.; Mutschler, I.; Aertsen, A.; Schulze-Bonhage, A. Signal quality of simultaneously recorded invasive and non-invasive EEG. Neuroimage 2009, 46, 708-716, doi:10.1016/j.neuroimage.2009.02.028.

4. Rubehn, B.; Bosman, C.; Oostenveld, R.; Fries, P.; Stieglitz, T. A MEMS-based flexible multichannel ECoGelectrode array. J. Neural Eng. 2009, 6, 36003, doi:10.1088/1741-2560/6/3/036003.

5. Kim, D.-H.; Viventi, J.; Amsden, J.J.; Xiao, J.; Vigeland, L.; Kim, Y.-S.; Blanco, J.A.; Panilaitis, B.; Frechette, E.S.; Contreras, D.; et al. Dissolvable films of silk fibroin for ultrathin conformal bio-integrated electronics. Nat. Mater. 2010, 9, 511-517, doi:10.1038/nmat2745.

6. Tolstosheeva, E.; Gordillo-González, V.; Biefeld, V.; Kempen, L.; Mandon, S.; Kreiter, A.K.; Lang, W. A multi-channel, flex-rigid ECoG microelectrode array for visual cortical interfacing. Sensors 2015, 15, 832854, doi:10.3390/s150100832. 
7. Takahashi, H.; Ejiri, T.; Nakao, M.; Nakamura, N.; Kaga, K.; Hervé, T. Microelectrode array on folding polyimide ribbon for epidural mapping of functional evoked potentials. IEEE Trans. Biomed. Eng. 2003, 50, 510-516, doi:10.1109/TBME.2003.809483.

8. Rousche, P.J.; Pellinen, D.S.; Pivin, D.P.; Williams, J.C.; Vetter, R.J.; Kipke, D.R. Flexible polyimide-based intracortical electrode arrays with bioactive capability. IEEE Trans. Biomed. Eng. 2001, 48, 361-371, doi:10.1109/10.914800.

9. Morris, S.; Hirata, M.; Sugata, H.; Goto, T.; Matsushita, K.; Yanagisawa, T.; Saitoh, Y.; Kishima, H.; Yoshimine, T. Patient-specific cortical electrodes for sulcal and gyral implantation. IEEE Trans. Biomed. Eng. 2015, 62, 1034-1041, doi:10.1109/TBME.2014.2329812.

10. Larmagnac, A.; Eggenberger, S.; Janossy, H.; Vörös, J. Stretchable electronics based on Ag-PDMS composites. Sci. Rep. 2014, 4, 7254, doi:10.1038/srep07254.

11. Stieghorst, J.; Tran, B.N.; Hadeler, S.; Beckmann, D.; Doll, T. Hydrogel-Based Actuation for Modiolar Hugging Cochlear Implant Electrode Arrays. Procedia Eng. 2016, 168, 1529-1532, doi:10.1016/j.proeng.2016.11.453.

12. Nahvi, M.S.; Boroumand, F.A.; Maghami, M.H.; Sodagar, A.M.; Shojaei, A.; Mirnajafi-Zadeh, J. Design, fabrication, and test of flexible thin-film microelectrode arrays for neural interfaces. In Proceedings of the 2017 IEEE 30th Canadian Conference on Electrical and Computer Engineering (CCECE), Windsor, ON, Canada, 30 April-3 May 2017; pp 1-4, ISBN 978-1-5090-5538-8.

13. Yan, Z.; Pan, T.; Xue, M.; Chen, C.; Cui, Y.; Yao, G.; Huang, L.; Liao, F.; Jing, W.; Zhang, H.; et al. Thermal Release Transfer Printing for Stretchable Conformal Bioelectronics. Adv. Sci. 2017, 4, 1700251, doi:10.1002/advs.201700251.

(C) 2020 by the authors. Submitted for possible open access publication under the terms and conditions of the Creative Commons Attribution (CC BY) license (http://creativecommons.org/licenses/by/4.0/). 